\title{
Validity of two short screeners for diet quality in time-limited settings
}

\author{
Helmut Schröder ${ }^{1,2, *}$, Alejandra Benitez Arciniega ${ }^{1,2}$, Cristina Soler ${ }^{3}$, \\ Maria-lsabel Covas ${ }^{1,2}$, José Miguel Baena-Díez ${ }^{4}$ and Jaume Marrugat ${ }^{3}$ \\ on behalf of the REGICOR and HERMES investigators $†$ \\ ${ }^{1}$ Cardiovascular Risk and Nutrition Research Group (CARIN-ULEC), Program of Research in Inflammatory and \\ Cardiovascular Disorders (RICAD), IMIM - Hospital del Mar, Biomedical Research Park, c/Doctor Aiguader 88, \\ 08003 Barcelona, Spain: ${ }^{2}$ Network of Centers for Biomedical Research (Centros de Investigación Biomédica en \\ Red - CIBER), Physiopathology of Obesity and Nutrition (CIBEROBN), Instituto de Salud Carlos III, Madrid, \\ Spain: ${ }^{3}$ Cardiovascular Epidemiology and Genetics Research Group (EGEC-ULEC), Program of Research in \\ Inflammatory and Cardiovascular Disorders (RICAD), IMIM, Barcelona, Spain: ${ }^{4}$ Centro de Salud La Marina; \\ IDIAP Jordi Gol, Barcelona, Spain
}

Submitted 18 October 2010: Accepted 14 June 2011: First published online 23 August 2011

\begin{abstract}
Objective: An urgent need in dietary assessment is the development of short tools that provide valid assessments of dietary quality for use in time-limited settings. The present study assessed concurrent and construct validity of the short Diet Quality Screener (sDQS) and brief Mediterranean Diet Screener (bMDSC) questionnaires. Design: Relative validity was measured by comparing three dietary quality indices the Diet Quality Index (DQI), the modified Mediterranean Diet Score (mMDS) and the Antioxidant Score (ANTOX-S) - derived from the two questionnaires with those from multiple $24 \mathrm{~h}$ recalls over 12 months. Construct validity was demonstrated by correlations between average nutrient intake recorded on multiple $24 \mathrm{~h}$ recalls and the DQI, mMDS and ANTOX-S derived by the short screeners.

Setting: Both short questionnaires were administered to 102 participants recruited from a population-based survey in Spain.

Results: DQI, mMDS and ANTOX-S correlated $(P<0 \cdot 001)$ with the corresponding $24 \mathrm{~h}$ recall indices $(r=0 \cdot 61,0 \cdot 40$ and $0 \cdot 45$, respectively). Limits of agreement lay between 96 and 126\%, 59 and 144\% and 61 and 118\% for the DQI, ANTOX-S and mMDS, respectively. Dietary intakes of fibre, vitamin $\mathrm{C}$, vitamin $\mathrm{E}, \mathrm{Mg}$ and $\mathrm{K}$ reported on the $24 \mathrm{~h}$ recalls were positively associated $(P<0.04)$ with the DQI, mMDS and ANTOX-S indices.

Conclusions: The sDQS and bMDSC provide reasonable approximations to food-based dietary indices and accurately situate subjects within the indices constructed for the present validation study.
\end{abstract}

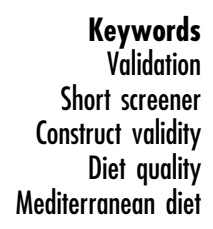

Non-communicable diseases such as CVD and cancer are estimated to be responsible for $60 \%$ of world deaths, and dietary habits appear to be strong determinants for their development ${ }^{(1)}$. Therefore, analysis of dietary habits in large populations to identify dietary deficiencies is of paramount importance. FFQ are widely used to estimate food intake in large epidemiological settings. However, full-length FFQ are time consuming for participants and thus are unsuitable for routine clinical use and for non-dietary studies incorporating a broad spectrum of measurements. For this reason, several brief screening

$\dagger$ A full roster of REGICOR investigators and collaborators can be found at www.REGICOR.org/regicor.inv tools have been developed to assess intakes of major food groups. Most of these short dietary questionnaires focus on one or two dietary components ${ }^{(2-7)}$. Only a few brief screeners assess a broader range of dietary intake ${ }^{(8,9)}$, an approach that might help to identify subjects at nutritional risk by indicating their level of adherence to diet quality recommendations. To address the need for a brief, effective instrument, we designed short dietary screeners for two different settings. The brief Mediterranean Diet Screener (bMDSC) is intended to assess adherence to the healthy Mediterranean dietary pattern. The short Diet Quality Screener (sDQS) was created to estimate overall diet quality in primary-care settings. We calculated a modified Mediterranean Diet Score (mMDS) and an 
Antioxidant Score (ANTOX-S) from the bMDSC and a Diet Quality Index (DQI) from the sDQS. The rationale for constructing the mMDS, ANTOX-S and DQI was adherence to the Mediterranean diet, antioxidant capacity of foods and dietary recommendations for the Spanish population, respectively ${ }^{(10,11)}$. The objective of the present study was first to analyse the degree to which the dietary indices derived from the bMDSC and SDQI questionnaires correlate with those obtained by a reference method (concurrent validity) and second to determine the ability of the screeners to measure diet quality (construct validity) in a random subsample of a population-based cohort in Spain.

\section{Material and methods}

\section{Study participants}

The validation study consecutively selected 150 men and women from a population-based cross-sectional survey performed in Girona Province between 2004 and 2006. That survey of 6352 randomly selected free-living men and women, aged 3 to 80 years ( $71.5 \%$ response rate), obtained sociodemographic, anthropometric and lifestyle variables including diet from all participants. Of those initially recruited, 133 agreed to participate in the validation study and 102 completed both short dietary screeners and a $24 \mathrm{~h}$ food recall questionnaire on at least ten occasions. The study was approved by the institutional ethics committee (Comités Éticos de Investigación Clínica-Instituto Municipal de Asistencia Sanitaria, Barcelona, Spain), participants signed an informed consent, and results of the examination were sent to all participants.

\section{Measurement of non-dietary variables}

Information on demographic and socio-economic variables, co-morbidity history, diet and lifestyle factors, including tobacco smoking and alcohol consumption, was obtained through structured standard questionnaires administered by trained personnel ${ }^{(12)}$.

Leisure-time physical activity was measured by the Minnesota leisure-time physical activity questionnaire, also administered by a trained interviewer ${ }^{(13,14)}$.

\section{Dietary assessment instruments}

\section{Multiple $24 \mathrm{~b}$ recalls (reference method)}

Monthly $24 \mathrm{~h}$ recalls were collected by telephone over a 12-month period by a trained interviewer and data from at least ten recalls were required for inclusion in the analysis. Dietary recalls were conducted on nonconsecutive days, including at least five weekdays and one weekend day, and participants were not alerted in any way to the date when they would be interviewed. Food intake data recorded during the $24 \mathrm{~h}$ recalls were grouped into the food-based dietary components of the bMDSC and sDQS for analysis.
We validated the dietary quality estimates from the bMDSC and sDQS by comparing them with the data gathered from the $24 \mathrm{~h}$ recalls.

\section{The brief Mediterranean diet screener}

The bMDSC was originally developed for another study $^{(15)}$. The objective of this screener was to calculate adherence to the Mediterranean diet and to qualitatively estimate dietary antioxidant intake. For this reason we included food items considered characteristic for the Mediterranean diet and food items with high antioxidant capacity. After participants had completed the multiple $24 \mathrm{~h}$ recalls they received the bMDSC by postal mail, together with short instructions on completion of the screener. Participants were asked to report their consumption frequency of fifteen selected food items during the preceding year: (i) pulses; (ii) green leafy and cabbage-like vegetables; (iii) other vegetables; (iv) red meat, sausages and cold cuts; (v) white meat; (vi) blue fish; (vii) white fish; (viii) dairy products; (ix) citrus fruits and berries; (x) other fruits; (xi) wholegrain products; (xii) olive oil; (xiii) nuts; (xiv) juice; (xv) red wine. The eight frequency categories range from 'never' to 'more than 4 times a day'. Standardized portion sizes were used to quantify food intake ${ }^{(16,17)}$.

\section{The short diet quality screener}

The sDQS was developed for use in primary-care settings to estimate overall diet quality. Within 1 week of returning the completed bMDSC, the participants received the sDQS, which was mailed to them with brief instructions on completion of the screener. Subjects were asked to base their responses on their usual dietary behaviours over the previous 12 months, reporting their habitual intake of eighteen food items grouped in three food categories. These categories were based on recommended frequencies of food intake ${ }^{(11)}$. Standardized portion sizes were used to quantify frequency of food intake $^{(16,17)}$. The first category includes eight items: (i) bread; (ii) vegetables (cooked and raw); (iii) fruit; (iv) milk and yoghurt; (v) rice and pasta; (vi) vegetable oils (olive and sunflower); (vii) alcoholic beverages; and (viii) cereals (cornflakes, muesli, etc.). The second category includes seven items: (i) meat; (ii) sausages; (iii) cheese; (iv) pastry; (v) animal fat (butter, lard); (vi) other vegetable oils (palm oil, etc.); and (vii) fast food. The third category had just three items: (i) fish; (ii) legumes; and (iii) nuts.

Food frequency consumption was arranged in three frequency response categories: (i) 'less than once a day', 'once a day' and 'more than once a day' for the eight food items in the first category; (ii) 'less than 4 times a week', ' 4 to 6 times a week' and 'once a day' for the seven food items in the second category; and (iii) 'less than 2 times a week', ' 2 to 3 times a week' and ' 4 or more times a week' for the three food items in the third food category. 
The aim of the study was to validate the scores by analysis of the corresponding food items. Therefore, food items listed in the $24 \mathrm{~h}$ recall that were not part of the scores obtained by the screeners were omitted.

\section{Calculation of dietary scores}

The antioxidant food and modified Mediterranean diet scores derived from the brief Mediterranean diet screener

The bMDSC provides two sub-scores: the antioxidant food score (ANTOX-S) and the modified Mediterranean diet score (mMDS; Table 1). The standardized portion size of each food group was multiplied by the corresponding frequency response category to obtain the amount of intake in $\mathrm{g} / \mathrm{d}$. Tertile distribution of food items included in the ANTOX-S and mMDS was calculated. The ANTOX-S was calculated by assigning 1 point for lowest tertile ratings, 2 points for medium tertile ratings and 3 points for highest tertile ratings for the following food items: (i) citrus fruits; (ii) other fruits; (iii) green leafy vegetables; (iv) other vegetables; (v) fruit juice; and (vii) red wine. The mMDS includes ten food items: (i) vegetables (green leafy vegetables and other vegetables); (ii) fruits (citrus fruits and other fruits); (iii) dairy products; (iv) red meat and sausages; (v) wholegrain products; (vi) fish; (vii) legumes; (viii) nuts; (ix) red wine; and (x) olive oil. Tertile ratings for vegetables, fruits, wholegrain products, legumes, nuts and olive oil were scored as mentioned above. Tertile distribution ratings of two food groups (dairy products; red meat and sausages) were coded inversely (first tertile 3 points, second tertile 2 points, and third tertile 1 point) because these foods are not considered part of the Mediterranean diet. Moderate red wine consumption (1-2 glasses/d) was coded as 3 and included as a favourable component in the score; no red wine consumption and more than 2 glasses/d were both coded as 1 . These values for the food items were added together to determine the ANTOX-S and mMDS, which range from 6 (very low adherence) to 18 (optimal adherence), and from 10 (very low adherence) to 30 (optimal adherence), respectively.

\section{The diet quality index derived from the short}

diet quality screener

The diet quality index (DQI) includes three food group categories (Table 2): with the exception of alcoholic beverage consumption, daily intake of 1 portion of foods in the first food group category is scored 2; lower and higher intakes are scored 1 and 3, respectively. Daily consumption of one alcoholic drink ( 1 bottle of beer, 1 glass of wine, or 1 cup of liquor equivalent to approximately $12 \mathrm{~g}$ of alcohol) is scored 3; lower and higher intakes are scored 1. Consumption of foods considered detrimental in the second food group category is scored 2 if reported as 4-6 times per week; more and less frequent consumption are scored 1 and 3, respectively. High consumption (4 or more times per week) of food items considered beneficial of the third food group category is scored 3. Intakes of 2-3 times and less than twice a week are scored 2 and 1, respectively. All food item scores are added up. The total possible score ranges from 18 to 54 .

\section{Statistical analyses}

Differences in continuous variables with normal distribution were compared between participant and non-participant groups using Student's $t$ test; the Mann-Whitney $U$ test was used otherwise. The $\chi^{2}$ test was used for categorical variables. Relative agreement of the dietary quality indices,

Table 1 Scoring method for the modified Mediterranean Diet Score (mMDS) and the Antioxidant Score (ANTOX-S)

\begin{tabular}{|c|c|c|c|c|}
\hline & & \multicolumn{3}{|c|}{ Scoring by tertile distribution of food } \\
\hline & & 1st tertile & 2nd tertile & 3rd tertile \\
\hline Pulses (beans, peas and lentils)* & 1 serving & 1 & 2 & 3 \\
\hline Green leafy and cabbage-like vegetables (lettuce, endive, broccoli, etc.)* $† \ddagger$ & 1 serving & 1 & 2 & 3 \\
\hline Other vegetables* ${ }^{*} \ddagger$ & 1 serving & 1 & 2 & 3 \\
\hline Red meat, sausages, cold cuts (pork, beef, lamb, salami, bratwurst, etc.) ${ }^{*}$ & 1 serving & 3 & 2 & 1 \\
\hline White meat (poultry and rabbit) & 1 serving & 3 & 2 & 1 \\
\hline Blue fish (tuna, sardine, salmon, etc. ${ }^{\star} \S$ & 1 serving & 1 & 2 & 3 \\
\hline Other fish (codfish, sole, flounder, etc.) ${ }^{*} \S$ & 1 serving & 1 & 2 & 3 \\
\hline Dairy products (cheese, whole milk, etc.)* & 1 serving & 3 & 2 & 1 \\
\hline Citrus fruits and berries (oranges, lemons, kiwis, strawberries, etc.) ${ }^{\star}+\|$ & 1 piece or 1 serving & 1 & 2 & 3 \\
\hline Other fruits* $+\|$ & 1 piece or 1 serving & 1 & 2 & 3 \\
\hline Wholegrain products* & 1 serving & 1 & 2 & 3 \\
\hline Olive oil* & 1 tablespoon & 1 & 2 & 3 \\
\hline Nuts (almonds, walnuts, etc.)* & 1 serving & 1 & 2 & 3 \\
\hline Juicet & 1 cup & 1 & 2 & 3 \\
\hline Red wine* $t$ & 1 cup & 1 & 2 & 3 \\
\hline
\end{tabular}

*Included in the mMDS.

tIncluded in the ANTOX-S.

¥Combined in the mMDS 'vegetable' food group.

§Combined in the mMDS 'fish' food group.

ICombined in the mMDS 'fruit' group. 
Table 2 Scoring method for the Diet Quality Index (DQI)

\begin{tabular}{|c|c|c|c|c|}
\hline \multicolumn{5}{|c|}{ 1. Daily frequency consumption of the following foods during the last 12 months } \\
\hline Food & Amount & $<1$ time/d & 1 time/d & $\geq 2$ times/d \\
\hline Bread & 1-2 slices & 1 & 2 & 3 \\
\hline Vegetable/salad & 1 serving & 1 & 2 & 3 \\
\hline Fruit & 1 piece or serving & 1 & 2 & 3 \\
\hline Yoghurt or milk & 1 tub/ 1 glass & 1 & 2 & 3 \\
\hline Pasta or rice & 1 serving & 1 & 2 & 3 \\
\hline Oil (olive or sunflower) & 1 tablespoon & 1 & 2 & 3 \\
\hline Alcoholic beverages & 1 drink & 1 & 3 & 1 \\
\hline Breakfast flakes & 1 bowl & 1 & 2 & 3 \\
\hline
\end{tabular}

2. Weekly frequency consumption of the following foods during the last 12 months

$\begin{array}{llccc}\text { Food } & \text { Amount } & <4 \text { times/week } & 4-6 \text { times/week } & 2 \\ \text { Meat } & 1 \text { serving } & 3 & 2 & 1 \\ \text { Sausages } & 1-3 \text { slices } & 3 & 2 & 1 \\ \text { Cheese } & 1 \text { serving } & 3 & 2 & 1 \\ \text { Pastry or sweets } & 1 \text { piece or serving } & 3 & 2 & 1 \\ \text { Butter or lard } & 1 \text { teaspoon } & 3 & 2 & 1 \\ \text { Other vegetable oils } & 1 \text { tablespoon } & 3 & 2 & 1 \\ \text { Fast food } & 1 \text { serving } & 3 & 1\end{array}$

3. Weekly frequency consumption of the following foods during the last 12 months

$\begin{array}{llccc}\text { Food } & \text { Amount } & <2 \text { times/week } & 2-3 \text { times/week } & 2 \\ \text { Fish } & 1 \text { serving } & 1 & 2 & 3 \\ \text { Legumes } & 1 \text { serving } & 1 & 2 & 3 \\ \text { Nuts } & 1 \text { handful } & 1 & 2 & 3\end{array}$

indicating the agreement between test and reference method in the ranking of subjects, was assessed by calculating Pearson product-moment correlation coefficients to compare the $24 \mathrm{~h}$ recall scores (reference method) with the subject's scores on the short screeners (test method). In addition, cross-classification models were fitted to test for gross misclassification of rating for the DQI, mMDS and ANTOX-S obtained from the test $v$. the reference method. Gross misclassification was defined as classification in the opposite tertile (lowest and highest) by the test method compared with the reference method. The proportions of correctly (i.e. similarly) categorized participants were also calculated.

Absolute agreement between scores derived from the short screeners and the $24 \mathrm{~h}$ recalls was analysed by the intra-class correlation coefficient (ICC). Additionally, we applied the limits of agreement (LOA) $\operatorname{method}^{(18)}$ expressed as a percentage. A mean agreement (difference of means) of $100 \%$ indicates total agreement between the test and reference method. For each score we plotted the difference between methods against the mean of the two. Linear regression analysis of mean differences of the dietary indices, as the dependent variable, and the mean of the corresponding dietary indices obtained by the test and the reference, as the independent variable, was performed to detect proportional biases (i.e. mean difference was significantly changed by the magnitude ratings for dietary indices). We applied the criteria for a reasonable agreement between estimates according to Ambrosini and colleagues ${ }^{(19)}$ : (i) LOA of the dietary indices derived from the test method were between one-half (50\%) and twice $(200 \%)$ their reference method estimate; and (ii) there were no significant changes of mean differences over the range of average ratings for the dietary indices.

To analyse construct validity, we hypothesized a relationship between a higher scoring for the dietary indices obtained by the short screeners and a more favourable nutrient intake profile reported on the $24 \mathrm{~h}$ recalls. General linear modelling procedures (GLM) were used to estimate nutrient intakes according to the tertile distribution of the dietary indices (DQI, mMDS and ANTOX-S). Linear trend was tested by including the categorized variable (tertile distribution of the dietary indices as continuous in this model). The polynomial contrast was used to determine $P$ for linear trend for continuous variables and a post boc Bonferroni correction for multiple comparisons was carried out. Differences were considered significant if $P<0 \cdot 05$. The SPSS for Windows statistical software package version 15 (SPSS, Inc., Chicago, IL, USA) was used to carry out all statistical analyses.

\section{Results}

With the exception of educational level, no statistically significant differences were observed between the main characteristics of participants included in the validation study and the remaining participants of the populationbased survey (Table 3). The DQI, mMDS and ANTOX-S were normally distributed. 
Table 3 Characteristics of the validation study participants and the remaining participants of the population-based cross-sectional survey ${ }^{*}$

\begin{tabular}{|c|c|c|c|c|c|}
\hline & \multicolumn{2}{|c|}{$\begin{array}{c}\text { Validation study } \\
\text { participants ( } n \text { 102) }\end{array}$} & \multicolumn{2}{|c|}{ Non-participants (n 6250) } & \multirow[b]{2}{*}{$P$ value } \\
\hline & Mean or median & $\begin{array}{l}\text { sD, } 95 \% \mathrm{Cl} \text { or } \\
\text { P25-P75 }\end{array}$ & Mean or median & $\begin{array}{l}\text { sD, } 95 \% \mathrm{Cl} \text { or } \\
\text { P25-P75 }\end{array}$ & \\
\hline Age (years) & $58 \cdot 6$ & $12 \cdot 1$ & $56 \cdot 6$ & $12 \cdot 6$ & $0 \cdot 119$ \\
\hline Sex (\% women) & $49 \cdot 0$ & $39 \cdot 3,58 \cdot 7$ & $52 \cdot 3$ & $39 \cdot 3,58 \cdot 7$ & $0 \cdot 487$ \\
\hline Leisure-time physical activity $(\mathrm{MET} \times \mathrm{min} / \mathrm{d})$ & 263 & $157-417$ & 231 & $119-402$ & $0 \cdot 233$ \\
\hline BMI $\left(\mathrm{kg} / \mathrm{m}^{2}\right)$ & $27 \cdot 6$ & $4 \cdot 2$ & $27 \cdot 4$ & $5 \cdot 1$ & 0.562 \\
\hline Obesity (\%)† & $26 \cdot 7$ & $18 \cdot 8,35 \cdot 1$ & $24 \cdot 4$ & $23 \cdot 3,25 \cdot 5$ & 0.587 \\
\hline Education higher than primary school (\%) & $62 \cdot 7$ & $53 \cdot 0,72 \cdot 4$ & $50 \cdot 0$ & $48 \cdot 8,51 \cdot 3$ & $0 \cdot 011$ \\
\hline Low energy reporter (\%)‡ & $17 \cdot 8$ & $10 \cdot 1,25 \cdot 5$ & $19 \cdot 3$ & $18 \cdot 3,20 \cdot 3$ & 0.707 \\
\hline
\end{tabular}

P25, 25th percentile; P75, 75th percentile; MET, metabolic equivalent task.

${ }^{*}$ Categorical variables are presented as relative frequency $(95 \% \mathrm{Cl})$; continuous variables are presented as mean or median (sD or P25-P75). tBMI $\geq 30 \cdot 0 \mathrm{~kg} / \mathrm{m}^{2}$.

‡Ratio of energy intake (reported on a validated full-length FFQ) to BMR of $<1 \cdot 2$.

Table 4 Correlation coefficients and between-method agreement measurements of dietary quality indices derived from the short Diet Quality Screener (sDQS) and the brief Mediterranean Diet Score Screener (bMDSS) with the reference method (24h recall)

\begin{tabular}{|c|c|c|c|c|c|c|}
\hline & \multicolumn{2}{|r|}{ sDQS } & \multicolumn{4}{|c|}{ bMDSC } \\
\hline & \multicolumn{2}{|r|}{ DQI } & \multicolumn{2}{|r|}{ mMDS } & \multicolumn{2}{|c|}{ ANTOX-S } \\
\hline & Mean & sD or $95 \% \mathrm{Cl}$ & Mean & sD or $95 \% \mathrm{Cl}$ & Mean & sD or $95 \% \mathrm{Cl}$ \\
\hline Corresponding screener & $39 \cdot 3$ & $2 \cdot 8$ & $18 \cdot 3$ & $2 \cdot 7$ & $11 \cdot 0$ & $2 \cdot 1$ \\
\hline $24 \mathrm{~h}$ recall & $35 \cdot 5$ & $2 \cdot 8$ & $20 \cdot 7$ & $3 \cdot 0$ & $10 \cdot 9$ & $2 \cdot 1$ \\
\hline Difference of means* & $3 \cdot 82$ & $3 \cdot 33,4 \cdot 31$ & $-2 \cdot 44$ & $-3 \cdot 01,-1 \cdot 82$ & 0.05 & $-0.49,0.39$ \\
\hline Proportions of means $(\%) \dagger$ & 111 & 109,112 & 89 & 86,92 & 101 & 97,106 \\
\hline LOA (\%)‡ & $96 ; 126$ & & $61 ; 118$ & & $59 ; 144$ & \\
\hline Regression coefficient§ & 0.040 & $-0 \cdot 162,0 \cdot 242$ & -0.200 & $-0.503,0 \cdot 103$ & 0.061 & $-0 \cdot 270,0.392$ \\
\hline ICC $\|$ & & 0.32 & & $0 \cdot 30$ & & 0.45 \\
\hline (screener $v .24 \mathrm{~h}$ recall) & & 0.61 & & $0 \cdot 40$ & & 0.45 \\
\hline Same tertile (\%) (screener v. $24 \mathrm{~h}$ recall) & & $48 \cdot 5$ & & $44 \cdot 0$ & & $50 \cdot 0$ \\
\hline Opposite tertile (\%) (screener $v .24 \mathrm{~h}$ recall) & & $3 \cdot 9$ & & $11 \cdot 0$ & & $9 \cdot 0$ \\
\hline
\end{tabular}

DQI, Dietary Quality Index; ANTOX-S, Antioxidant Score; mMDS, modified Mediterranean Diet Score.

One hundred and two participants recruited from a population-based survey in Spain were administered both short questionnaires and completed at least ten interviewer-administered $24 \mathrm{~h}$ recalls over a 12 -month period.

${ }^{*}$ Calculated as: screener $-24 \mathrm{~h}$ recall.

tCalculated as: (screener/ $24 \mathrm{~h}$ recall) $\times 100$.

$\$ 95 \%$ limits of agreement indicating lower and upper limits of agreement.

§Regression coefficient $(\beta)$ between mean (dependent variables) and mean differences (independent variables) of the dietary indices obtained by the test and the reference method.

IIIntra-class correlation coefficient.

-Pearson correlation coefficient.

The sDQS significantly overestimated the DQI rating, whereas the opposite finding was observed for the bMDSC, which underestimated the mMDS score (Table 4). Mean difference of the ANTOX-S was close to zero. The test methods' estimates for DQI and mMDS were 11\% higher and $11 \%$ lower than those of the $24 \mathrm{~h}$ recall reference method, respectively (Table 4). The range of LOA was highest for the ANTOX-S and lowest for the mMDS. No significant proportional variation of the agreement between the test and reference method was observed across ratings of the dietary indices (Table 4, Fig. 1).

The ICC between scores obtained by the screeners and the corresponding scores retrieved by the reference method was highest for the ANTOX-S followed by the DQI and mMDS.
Furthermore, Table 4 shows the Pearson correlation coefficients between methods, indicating the capacity of the bMDSC and the sDQS to rank subjects in accordance with their DQI, mMDS and ANTOX-S ratings. Additionally, gross misclassification models were fitted. Pearson correlation coefficients between scores (DQI, mMDS and ANTOX-S) retrieved from both screeners (sDQI and bMDSC) and the corresponding scores obtained from the reference method (multiple $24 \mathrm{~h}$ recalls) were calculated. All three dietary scores obtained from both short screeners were significantly correlated with the corresponding scores obtained using the reference method. The correlation coefficient was highest and gross misclassification was lowest for the DQI derived from the sDQS; misclassification was somewhat higher for mMDS and ANTOX-S ratings. 
(a)

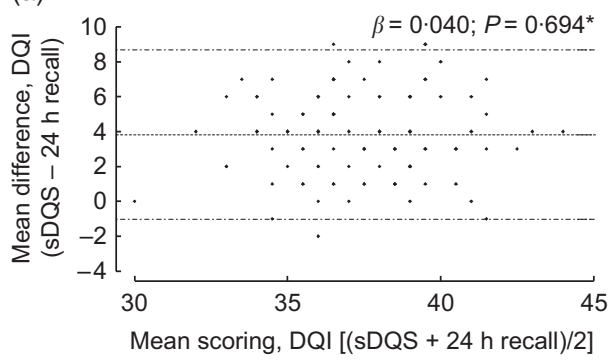

(b)

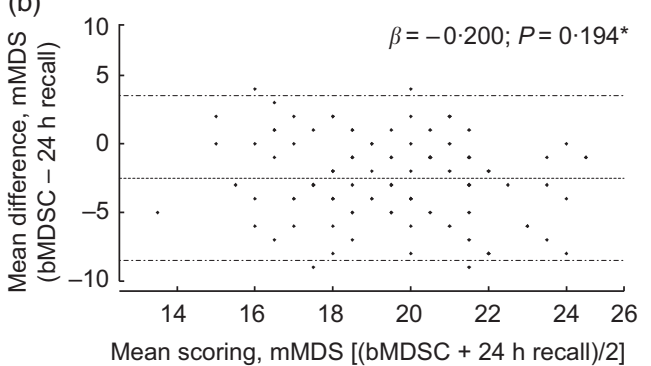

(c)

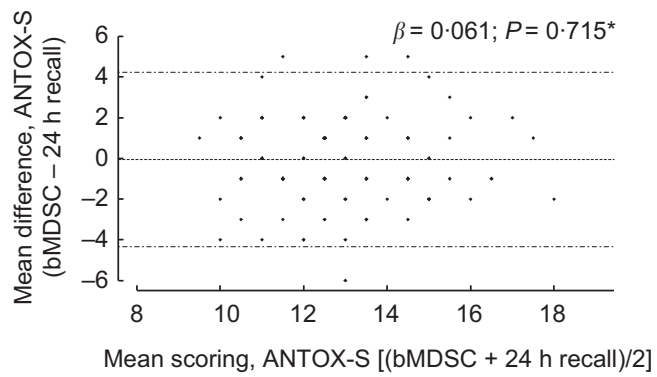

Fig. 1 Bland-Altman plots indicating the mean difference between indices obtained from the $24 \mathrm{~h}$ recall (reference method) and the corresponding dietary assessment method plotted $v$. the mean of the indices obtained from the two methods for: (a) the Diet Quality Index (DQI) derived from the short Dietary Quality Screener (sDQS); (b) the modified Mediterranean Diet Score (mMDS) derived from brief Mediterranean Diet Score Screener (bMDSC); and (c) the Antioxidant Score (ANTOX-S) derived from the sDQS. One hundred and two participants recruited from a population-based survey in Spain were administered both short questionnaires and completed at least ten interviewer-administered $24 \mathrm{~h}$ recalls over a 12 -month period. -..- indicates mean difference; -. - - - indicate upper and lower $95 \%$ limits of agreement. *Regression coefficient and statistical significance of the slope from linear regression of the mean of the methods $v$. the difference between methods

Table 5 shows the construct validity of the DQI, mMDS and ANTOX-S. As expected, a direct association existed between dietary fibre, vitamin $\mathrm{C}$, vitamin $\mathrm{E}, \mathrm{Mg}$ and $\mathrm{K}$ and the tertiles of all three dietary scores. The intakes of folic acid and flavonoids increased across tertile distributions of the DQI and ANTOX-S. In contrast, trans fatty acids and the ratio of saturated fat to unsaturated fat decreased with higher ratings for the DQI.

\section{Discussion}

The three food-based indices derived from the sDQS and the bMDSC reasonably ranked populations into similar score levels derived by $24 \mathrm{~h}$ recalls. Furthermore, the three dietary indices were positively associated with a beneficial nutrient intake profile.

Following a healthy diet is paramount for physical and mental health ${ }^{(1,20-22)}$. The prevention and treatment of non-communicable diseases such as diabetes, obesity and CVD is strongly influenced through diet ${ }^{(23)}$, and therefore identification of individuals at nutritional risk is important to public health policy and initiatives. However, lengthy comprehensive dietary assessment is a time-consuming process that becomes a real challenge in multidisciplinary epidemiological research and primary-care settings. The sDQS and bMDSC were created to rapidly capture diet quality based on food intake. The DQI, mMDS and ANTOX-S dietary indices derived from these questionnaires showed moderate to good correlations with the corresponding indices obtained by $24 \mathrm{~h}$ recalls. The observed range of Pearson correlation $(0 \cdot 40-0 \cdot 61)$ and the average level of gross misclassification $(7 \cdot 7 \%)$ are comparable to those reported for food and nutrient intakes derived from short diet screeners and full-length $\mathrm{FFQ}^{(24-28)}$. Although a slightly higher correlation has been reported between the Diet Quality Index-Revised (DQI-R) derived from a full-length FFQ and two 1-week dietary records ${ }^{(29)}$, our results suggest that the sDQS and bMDSC adequately rank subjects with respect to the dietary indices' ratings.

However, reasonable relative agreement between methods does not necessarily imply good absolute agreement. To address this issue we plotted mean differences between the dietary indices derived from the test and reference methods against the corresponding mean of both methods and calculated the LOA. The sDQS and the bMDSC systematically overestimated and underestimated the DQI and mMDS ratings, respectively. However, no significant proportional bias was observed for all three dietary indices. Furthermore, the widest range of LOA between methods was found for the ANTOX-S, meaning that $95 \%$ of all bMDSC estimates were between $59 \%$ (underestimating by $41 \%$ ) and $144 \%$ (overestimating by 
Table 5 Energy and nutrient intakes recorded on $24 \mathrm{~h}$ recalls according to tertile distribution of dietary indices derived from the short Dietary Quality Screener (sDQS) and brief Mediterranean Diet Score Screener (bMDSC)

\begin{tabular}{|c|c|c|c|c|c|c|c|}
\hline & \multicolumn{2}{|c|}{ First tertile } & \multicolumn{2}{|c|}{ Second tertile } & \multicolumn{2}{|c|}{ Third tertile } & \multirow[b]{2}{*}{$P$ for trenc } \\
\hline & Mean & $95 \% \mathrm{Cl}$ & Mean & $95 \% \mathrm{Cl}$ & Mean & $95 \% \mathrm{Cl}$ & \\
\hline \multicolumn{8}{|l|}{ Energy (MJ) } \\
\hline DQI & $7 \cdot 4$ & $6 \cdot 9,7 \cdot 9$ & $7 \cdot 2$ & $6 \cdot 6,7 \cdot 8$ & $7 \cdot 1$ & $6 \cdot 5,7 \cdot 1$ & 0.205 \\
\hline mMDS & $7 \cdot 0$ & $6 \cdot 5,7 \cdot 5$ & $7 \cdot 6$ & $7 \cdot 0,8 \cdot 2$ & $7 \cdot 2$ & $6 \cdot 6,7 \cdot 7$ & $0 \cdot 697$ \\
\hline ANTOX-S & $7 \cdot 2$ & $6 \cdot 7,7 \cdot 7$ & $7 \cdot 2$ & $6 \cdot 6,7 \cdot 7$ & $7 \cdot 3$ & $6 \cdot 6,8 \cdot 0$ & $0 \cdot 870$ \\
\hline \multicolumn{8}{|c|}{ Carbohydrates (\% of energy) } \\
\hline DQI & $40 \cdot 6$ & $38 \cdot 6,42 \cdot 6$ & $41 \cdot 9$ & $39 \cdot 6,44 \cdot 1$ & $42 \cdot 5$ & $40 \cdot 4,44 \cdot 7$ & 0.205 \\
\hline MMDS & $42 \cdot 4$ & $40 \cdot 9,44 \cdot 8$ & $39 \cdot 3$ & $37 \cdot 0,41 \cdot 5$ & $43 \cdot 0$ & $40 \cdot 9,45 \cdot 1$ & 0.670 \\
\hline ANTOX-S & $41 \cdot 6$ & $39 \cdot 7,43 \cdot 5$ & $41 \cdot 4$ & $39 \cdot 3,43 \cdot 4$ & $42 \cdot 4$ & $39 \cdot 7,45 \cdot 2$ & 0.632 \\
\hline \multicolumn{8}{|c|}{ Protein (\% of energy) } \\
\hline DQI & $16 \cdot 3$ & $15 \cdot 6,17 \cdot 0$ & $17 \cdot 1$ & $16 \cdot 3,18 \cdot 0$ & $17 \cdot 2$ & $16 \cdot 4,18 \cdot 0$ & 0.088 \\
\hline MMDS & $17 \cdot 2$ & $16 \cdot 5,17 \cdot 9$ & $16 \cdot 8$ & $15 \cdot 9,17 \cdot 6$ & $16 \cdot 5$ & $15 \cdot 7,17 \cdot 3$ & $0 \cdot 196$ \\
\hline ANTOX-S & $17 \cdot 3$ & $16 \cdot 6,18 \cdot 0$ & $16 \cdot 5$ & $15 \cdot 9,17 \cdot 3$ & $16 \cdot 5$ & $15 \cdot 5,17 \cdot 5$ & $0 \cdot 163$ \\
\hline \multicolumn{8}{|l|}{ Fat $(\%)$} \\
\hline DQI & $43 \cdot 8$ & $42 \cdot 3,45 \cdot 5$ & $43 \cdot 3$ & $41 \cdot 6,45 \cdot 1$ & $41 \cdot 5$ & $39 \cdot 7,43 \cdot 2$ & 0.047 \\
\hline MMDS & $42 \cdot 9$ & $41 \cdot 3,44 \cdot 5$ & $44 \cdot 1$ & $42 \cdot 1,45 \cdot 9$ & $41 \cdot 8$ & $40 \cdot 1,44 \cdot 0$ & $0 \cdot 378$ \\
\hline ANTOX-S & $43 \cdot 1$ & $41 \cdot 6,44 \cdot 7$ & $42 \cdot 8$ & $41 \cdot 4,44 \cdot 5$ & $42 \cdot 5$ & $40 \cdot 3,44 \cdot 7$ & 0.664 \\
\hline \multicolumn{8}{|c|}{ Ratio of saturated to unsaturated fat } \\
\hline DQI & 0.51 & $0.48,0.54$ & 0.47 & $0.44,0.50$ & 0.45 & $0 \cdot 42,0 \cdot 48$ & 0.008 \\
\hline MMDS & 0.47 & $0.44,0.51$ & 0.50 & $0.46,0.53$ & 0.47 & $0.44,0.50$ & $0 \cdot 790$ \\
\hline ANTOX-S & 0.48 & $0.45,0.51$ & 0.47 & $0.43,0.51$ & 0.49 & $0.45,0.52$ & $0 \cdot 854$ \\
\hline \multicolumn{8}{|c|}{ Total cholesterol (mg) } \\
\hline DQI & 290 & 263,317 & 277 & 247,308 & 253 & 224,283 & 0.007 \\
\hline MMDS & 283 & 256,310 & 296 & 265,327 & 248 & 219,277 & 0.087 \\
\hline ANTOX-S & 290 & 264,316 & 265 & 237,293 & 262 & 225,299 & $0 \cdot 224$ \\
\hline \multicolumn{8}{|c|}{ Trans fatty acids (g) } \\
\hline DQI & $1 \cdot 07$ & $0 \cdot 82,1 \cdot 31$ & $0 \cdot 73$ & $0 \cdot 46,1 \cdot 0$ & 0.67 & $0.41,0.93$ & 0.031 \\
\hline MMDS & 0.92 & $0 \cdot 67,1 \cdot 17$ & 0.87 & $0 \cdot 58,1 \cdot 16$ & $0 \cdot 71$ & $0.44,0.98$ & $0 \cdot 262$ \\
\hline ANTOX-S & $1 \cdot 0$ & $0 \cdot 78,1 \cdot 24$ & 0.83 & $0.57,1.08$ & $0 \cdot 50$ & $0 \cdot 15,0 \cdot 83$ & $0 \cdot 015$ \\
\hline \multicolumn{8}{|l|}{ Fibre (g) } \\
\hline DQI & $12 \cdot 9$ & $11 \cdot 5,14 \cdot 4$ & $15 \cdot 2$ & $13 \cdot 6,16 \cdot 4$ & $16 \cdot 9$ & $15 \cdot 3,18 \cdot 5$ & $<0.001$ \\
\hline MMDS & $13 \cdot 5$ & $12 \cdot 0,15 \cdot 0$ & $14 \cdot 0$ & $12 \cdot 3,15 \cdot 7$ & $17 \cdot 1$ & $15 \cdot 5,18 \cdot 7$ & $<0.001$ \\
\hline ANTOX-S & $13 \cdot 6$ & $12 \cdot 2,15 \cdot 0$ & $14 \cdot 7$ & $13 \cdot 2,16 \cdot 2$ & $17 \cdot 6$ & $15 \cdot 6,19 \cdot 6$ & $<0.001$ \\
\hline \multicolumn{8}{|c|}{ Vitamin C (mg) } \\
\hline DQI & $71 \cdot 6$ & $57 \cdot 6,85 \cdot 6$ & $110 \cdot 4$ & $94 \cdot 8,125 \cdot 9$ & $107 \cdot 6$ & $92 \cdot 5,122 \cdot 6$ & 0.001 \\
\hline MMDS & $80 \cdot 4$ & $65 \cdot 8,94 \cdot 9$ & $94 \cdot 0$ & $77 \cdot 3,110 \cdot 6$ & $109 \cdot 5$ & $93 \cdot 9,125 \cdot 1$ & 0.008 \\
\hline ANTOX-S & $72 \cdot 4$ & $60 \cdot 0,85 \cdot 0$ & $99 \cdot 1$ & $85 \cdot 4,112 \cdot 7$ & $129 \cdot 2$ & $111 \cdot 2,147 \cdot 1$ & $<0.001$ \\
\hline \multicolumn{8}{|c|}{ Vitamin E (mg) } \\
\hline DQI & $5 \cdot 4$ & $4 \cdot 8,5 \cdot 9$ & $6 \cdot 0$ & $5 \cdot 4,6 \cdot 7$ & $5 \cdot 9$ & $5 \cdot 3,6 \cdot 6$ & $0 \cdot 163$ \\
\hline MMDS & $5 \cdot 2$ & $4 \cdot 6,5 \cdot 8$ & $5 \cdot 8$ & $5 \cdot 2,6 \cdot 3$ & $6 \cdot 3$ & $5 \cdot 7,6 \cdot 9$ & 0.012 \\
\hline ANTOX-S & $5 \cdot 4$ & $4 \cdot 9,6 \cdot 0$ & $5 \cdot 7$ & $5 \cdot 1,6 \cdot 3$ & $6 \cdot 4$ & $5 \cdot 7,7 \cdot 2$ & 0.032 \\
\hline \multicolumn{8}{|l|}{ Folic acid $(\mu \mathrm{g})$} \\
\hline DQI & 184 & 168,200 & 225 & 207,242 & 226 & 209,243 & 0.001 \\
\hline MMDS & 198 & 181,215 & 208 & 189,228 & 221 & 203,239 & 0.067 \\
\hline ANTOX-S & 193 & 178,209 & 213 & 196,230 & 233 & 211,255 & 0.005 \\
\hline \multicolumn{8}{|l|}{$\mathrm{Mg}(\mathrm{mg})$} \\
\hline DQI & 206 & 190,221 & 232 & 216,249 & 248 & 232,264 & $<0.001$ \\
\hline mMDS & 207 & 192,223 & 228 & 210,245 & 246 & 230,263 & 0.001 \\
\hline ANTOX-S & 216 & 201,230 & 227 & 211,244 & 245 & 225,267 & 0.023 \\
\hline K (g) & & & & & & & \\
\hline DQI & $2 \cdot 3$ & $2 \cdot 1,2 \cdot 5$ & $2 \cdot 6$ & $2 \cdot 5,2 \cdot 8$ & $2 \cdot 7$ & $2 \cdot 5,2 \cdot 9$ & 0.001 \\
\hline MMDS & $2 \cdot 4$ & $2 \cdot 2,2 \cdot 5$ & $2 \cdot 5$ & $2 \cdot 3,2 \cdot 7$ & $2 \cdot 7$ & $2 \cdot 6,2 \cdot 9$ & 0.001 \\
\hline ANTOX-S & $2 \cdot 4$ & $2 \cdot 3,2 \cdot 6$ & $2 \cdot 5$ & $2 \cdot 4,2 \cdot 7$ & $2 \cdot 8$ & $2 \cdot 6,3 \cdot 0$ & 0.008 \\
\hline Flavonoids (g & & & & & & & \\
\hline DQI & $48 \cdot 8$ & $37 \cdot 4,60 \cdot 2$ & $70 \cdot 6$ & $58 \cdot 0,83 \cdot 1$ & $69 \cdot 0$ & $56 \cdot 5,80 \cdot 9$ & 0.020 \\
\hline MMDS & $53 \cdot 8$ & $42 \cdot 4,65 \cdot 1$ & $62 \cdot 2$ & $49 \cdot 2,75 \cdot 1$ & $66 \cdot 9$ & $54 \cdot 7,79 \cdot 0$ & $0 \cdot 121$ \\
\hline ANTOX-S & $49 \cdot 2$ & $39 \cdot 1,59 \cdot 2$ & $60 \cdot 7$ & $49 \cdot 7,71 \cdot 7$ & $60 \cdot 7$ & $69 \cdot 1,97 \cdot 9$ & $<0.001$ \\
\hline
\end{tabular}

DQI, Dietary Quality Index; mMDS, modified Mediterranean Diet Score; ANTOX-S, Antioxidant Score.

One hundred and two participants recruited from a population-based survey in Spain were administered both short questionnaires and completed at least ten interviewer-administered $24 \mathrm{~h}$ recalls over a 12-month period.

$44 \%)$ of their reference method estimates. These results indicate a reasonable absolute agreement between the dietary indices derived from the SDQS and the bMDSC and those of the reference method.
In addition to estimating the relative validity of both short dietary screeners studied, we sought to analyse their construct validity, i.e. the degree to which these dietary quality assessment tools measure the theoretical construct 
(diet quality) they intend to assess. For this purpose, we hypothesized that higher ratings on the dietary indices should be positively correlated with a healthy nutrient intake profile (i.e. higher intakes of vitamin C, vitamin E, dietary fibre, etc.) In most cases, the sDQS and bMDSC dietary indices correlated as anticipated with the average nutrient intakes derived from multiple $24 \mathrm{~h}$ recalls. A healthier nutrient intake profile was related to higher ratings of the DQI, mMDS and ANTOX-S, indicating a reasonable construct validity of the short dietary screeners. Construct validity was somewhat stronger for the sDQSderived DQI compared with the mMDS and ANTOX-S obtained from the bMDSC. The different food composition of both screeners might particularly explain this finding.

A limitation of the present study is the somewhat higher educational level of participants who completed the validation study.

We conclude that the sDQI and bMDSC accurately rate subjects with respect to the dietary indices derived from these screeners without significant proportional variations over the range of average ratings for the dietary indices. Additionally, the LOA variations are within a reasonable range. Furthermore, both screeners show reasonable construct validity, as indicated by the correlations between the dietary indices derived from the sDQS and bMDSC and nutrient intakes reported on the $24 \mathrm{~h}$ recalls. Hence, the sDQS and the bMDSC are valid dietary assessment tools for rapid estimation of dietary quality.

\section{Acknowledgements}

This research was supported by a grant 2FD097-0297CO2-01 from Fondo Europeo de Desarrollo Regional (FEDER) and by parts of grants from Spain's Ministerio de Sanidad y Consumo, Instituto de Salud Carlos III FEDER (PI080439), Red HERACLES RD06/0009, and by a joint contract of the Instituto de Salud Carlos III and the Health Department of the Catalan Government (Generalitat de Catalunya), CP 03/00115. The CIBEROBN is an initiative of the Instituto de Salud Carlos III, Madrid, Spain. The authors declare no conflict of interest. H.S. led data analysis and writing of the manuscript. J.M. and A.B.A. participated in the discussion and interpretation of results, and writing of the manuscript. J.M.B.-D., C.S. and M.-I.C. provided expertise in data analysis and in interpretation and discussion of results. They made substantial suggestions on the manuscript. All authors read and approved the final manuscript. The authors appreciate the English revision made by Elaine Lilly, PhD (Writers First Aid).

\section{References}

1. The World Health Report (2002) Reducing Risks, Promoting Healthy Life. Geneva: WHO.
2. Wiens L, Schulzer M, Chen C et al. (2010) Reliability and validity of the SmartDiet Canadian version questionnaire. I Am Diet Assoc 110, 101-105.

3. Spencer EH, Elon LK, Hertzberg VS et al. (2005) Validation of a brief diet survey instrument among medical students. J Am Diet Assoc 105, 802-806.

4. Cena H, Roggi \& Turconi G (2008) Development and validation of a brief food frequency questionnaire for dietary lutein and zeaxanthin intake assessment in Italian women. Eur J Nutr 47, 1-9.

5. Thompson FE, Midthune D, Subar AF et al. (2004) Performance of a short tool to assess dietary intakes of fruits and vegetables, percentage energy from fat and fibre. Public Health Nutr 7, 1097-1105.

6. Nelson MC \& Lytle LA (2009) Development and evaluation of a brief screener to estimate fast-food and beverage consumption among adolescents. J Am Diet Assoc 109, 730-734.

7. Thompson FE, Midthune D, Subar AF et al. (2007) Development and evaluation of a short instrument to estimate usual dietary intake of percentage energy from fat. J Am Diet Assoc 107, 760-767.

8. Mochari H, Gao Q \& Mosca L (2008) Validation of the MEDFICTS dietary assessment questionnaire in a diverse population. J Am Diet Assoc 108, 817-822.

9. Rifas-Shiman SL, Willett WC \& Lobb R (2001) PrimeScreen, a brief dietary screening tool: reproducibility and comparability with both a longer food frequency questionnaire and biomarkers. Public Health Nutr 4, 249-254.

10. Willett WC, Sacks F, Trichopoulou A et al. (1995) Mediterranean diet pyramid: a cultural model for healthy eating. Am J Clin Nutr 61, 6 Suppl, 1402S-1406S.

11. Aranceta $\mathrm{J} \&$ Serra-Majem L, Working Party for the Development of Food-Based Dietary Guidelines for the Spanish Population (2001) Dietary guidelines for the Spanish population. Public Health Nutr 4, 1403-1408.

12. Baena-Díez JM, Alzamora-Sas MT, Grau M et al. (2009) Validity of the MONICA cardiovascular questionnaire compared with clinical records. Gac Sanit 23, 519-525.

13. Elosua R, Garcia M, Aguilar A et al. (2000) Validation of the Minnesota Leisure Time Physical Activity Questionnaire in Spanish women. Investigators of the MARATDON Group. Med Sci Sports Exerc 32, 1431-1437.

14. Elosua R, Marrugat J, Molina L et al. (1994) Validation of the Minnesota Leisure Time Physical Activity Questionnaire in Spanish men. The MARATHOM Investigators. Am J Epidemiol 139, 1197-1209.

15. Peters A, Schneider A, Greven S et al. (2007) Air pollution and inflammatory response in myocardial infarction survivors: gene-environment interactions in a high-risk group. Inhal Toxicol 19, Suppl. 1, 161-175.

16. Serra Majem L (2004) Guia de Alimentación Saludable. Madrid: Sociedad Española de Nutrición Comunitaria.

17. Salas Salvado J, Bonada A, Tralerro R et al. (editors) (2000) Nutrición y Dietética Clinica. Barcelona: Editorial Masson.

18. Bland JM \& Altman DG (1986) Statistical methods for assessing agreement between two methods of clinical measurement. Lancet 1, 307-310.

19. Ambrosini GL, van Roosbroeck SA, Mackerras D et al. (2003) The reliability of ten-year dietary recall: implications for cancer research. J Nutr 133, 2663-2668.

20. Féart C, Samieri C \& Barberger-Gateau P (2010) Mediterranean diet and cognitive function in older adults. Curr Opin Clin Nutr Metab Care 13, 14-18.

21. Muñoz MA, Fíto M, Marrugat J et al. (2009) Adherence to the Mediterranean diet is associated with better mental and physical health. BrJ Nutr 101, 1821-1827.

22. Schroder H (2007) Protective mechanisms of the Mediterranean diet in obesity and type 2 diabetes. J Nutr Biochem 18, 149-160. 
23. World Health Organization (2003) Diet, Nutrition and the Prevention of Chronic Diseases. Report of a Joint WHO/FAO Expert Consultation. WHO Technical Report Series no. 916. Geneva: WHO.

24. Schroder H, Covas MI, Marrugat J et al. (2001) Use of a three-day estimated food record, a 72 -hour recall and a food-frequency questionnaire for dietary assessment in a Mediterranean Spanish population. Clin Nutr 20, 429-437.

25. Chan SG, Ho SC, Kreiger N et al. (2008) Validation of a food frequency questionnaire for assessing dietary soy isoflavone intake among midlife Chinese women in Hong Kong. J Nutr 138, 567-573.

26. Osowski JM, Beare T \& Specker B (2007) Validation of a food frequency questionnaire for assessment of calcium and bone-related nutrient intake in rural populations. $J \mathrm{Am}$ Diet Assoc 107, 1349-1355.

27. Sevak L, Mangtani P, McCormack V et al. (2004) Validation of a food frequency questionnaire to assess macro- and micro-nutrient intake among South Asians in the United Kingdom. Eur J Nutr 43, 160-168.

28. Fernández-Ballart JD, Piñol JL, Zazpe I et al. (2010) Relative validity of a semi-quantitative food-frequency questionnaire in an elderly Mediterranean population of Spain. BrJ Nutr 103, 1808-1816.

29. Newby PK, Hu FB, Rimm EB et al. (2003) Reproducibility and validity of the diet quality index revised as assessed by use of a food-frequency questionnaire. Am J Clin Nutr $\mathbf{7 8}$ 941-949. 